\title{
Prevalence and genetic characterization of Cryptosporidium, Enterocytozoon, Giardia and Cyclospora in diarrheal outpatients in china
}

\author{
Hua Liu ${ }^{1,2,3}$, Yujuan Shen ${ }^{1,2,3^{*}}$, Jianhai Yin ${ }^{1,2,3}$, Zhongying Yuan ${ }^{1,2,3}$, Yanyan Jiang ${ }^{1,2,3}$, Yuxin $X u^{1,2,3}$, Wei Pan ${ }^{1,2,3}$, \\ Yuan $\mathrm{Hu}^{1,2,3}$ and Jianping $\mathrm{CaO}^{1,2,3^{*}}$
}

\begin{abstract}
Background: Cryptosporidium spp., Enterocytozoon spp., Giardia spp. and Cyclospora spp. are important intestinal protozoan parasites causing diarrhea in humans, livestocks and wildlife and have a significant impact on public health. No reports exist about simultaneous prevalence rates or genotyping data of these four parasites in outpatients from China.

Methods: Fecal specimens from 252 diarrhea patients in a pediatric clinic $(n=169)$ and an intestinal clinic $(n=83)$ of a hospital in Shanghai, China, were collected between October 2012 and March 2013. All samples were examined for the presence of the four parasites by using molecular methods.

Results: In total, 76/252 (30.16\%) patients were positive for at least one intestinal parasite, of which Cryptosporidium spp., Enterocytozoon bieneusi and Giardia intestinalis were detected by nested PCR in 34 (13.49\%), 34 (13.49\%) and 17 (6.75\%) of the fecal specimens, respectively. Sequence analysis showed that all Cryptosporidium-positive specimens were $C$. andersoni and that most $G$. intestinalis- positive patients were infected by assemblage $C$, which is usually found in canids, while only one sample was from assemblage B. Eight patients were co-infected with Cryptosporidium spp. and Enterocytozoon, while one was co-infected with Cryptosporidium and Giardia.

Conclusions: The patients infected with Cryptosporidium and Enterocytozoon bieneusi had higher infection rates in winter than in spring in this area. Data indicated that C. andersoni is the fourth major Cryptosporidium species infecting humans in addition to C. hominis, C. parvum and C. meleagridis. Our study also revealed a short-term outbreak of cryptosporidiosis and microsporidiosis and sporadic cases of giardiasis that occurred among humans in Shanghai, China.
\end{abstract}

Keywords: Cryptosporidium, Enterocytozoon, Giardia, Cyclospora, Outpatients, Genotype

\section{Background}

Cryptosporidiosis, microsporidiosis, giardiasis and cyclosporiasis are emerging infectious diseases. Cryptosporidium spp., Enterocytozoon spp., Giardia spp. and Cyclospora spp. are diarrhea-causing intestinal protozoans of humans (especially in AIDS patients), livestock and wildlife worldwide [1-4]. Although they are considered opportunistic

\footnotetext{
* Correspondence: amyshyj12@163.com; caojp@yahoo.com
${ }^{1}$ National Institute of Parasitic Diseases, Chinese Center for Disease Control

*Correspondence: amyshyj12@163.com; Caojp@yahoo.com
'National Institute of Parasitic Diseases, Chinese Center for Disease Control and Prevention, Shanghai, People's Republic of China

${ }^{2}$ Key Laboratory of Parasite and Vector Biology, Ministry of Health, Shanghai, People's Republic of China

${ }^{3}$ WHO Collaborating Center for Malaria, Schistosomiasis and Filariasis, 'WHO Collaborating Center for Malaria,
Shanghai, People's Republic of China
}

\section{(O) Biomed Central}

(c) 2014 Liu et al.; licensee BioMed Central Ltd. This is an Open Access article distributed under the terms of the Creative Commons Attribution License (http://creativecommons.org/licenses/by/2.0), which permits unrestricted use, distribution, and reproduction in any medium, provided the original work is properly cited. The Creative Commons Public Domain Dedication waiver (http://creativecommons.org/publicdomain/zero/1.0/) applies to the data made available in this article, unless otherwise stated.

pathogens, they have caused several outbreaks and emergency responses among humans and animals [5-9] These four parasites pose significant challenges to public health and water authorities, especially in developing countries because of the high prevalence and disease burden of the infections $[10,11]$. Furthermore, they exert negative pressure on the growth and cognitive functions of infected children and immunocompromised persons $[1,12,13]$.

Cryptosporidium and microsporidia infect AIDS or immunodeficient patients, travelers, children and the elderly. C. parvum and C. hominis are the main humanpathogenic Cryptosporidium species, while $C$. muris and C. andersoni are minor zoonotic species, for which small 
numbers of human cases have recently been reported $[14,15]$. The prevalent microsporidia species E. bieneusi has been most frequently identified in human clinical fecal samples as well as in wild and domestic animals $[16,17]$. Molecular diagnostic tools have been used to trace the source of human infections and transmissions, thus confirming its zoonotic potential [18].

G. intestinalis is the etiologic agent of giardiasis, a common gastrointestinal disease in humans, livestock and companion animals. G. intestinalis is considered as a complex species and based on genetic analysis has been grouped into eight assemblages $(\mathrm{A}-\mathrm{H})[19,20]$. Both assemblages $\mathrm{A}$ and $\mathrm{B}$, which can be transmitted zoonotically, have a wide host range and are responsible for human infections [21,22]. Assemblages C-G appear to be strictly host-specific: $\mathrm{C}$ and $\mathrm{D}$ are found largely in canids, $\mathrm{E}$ in domestic mammals, $\mathrm{F}$ in cats, $\mathrm{G}$ in rodents and $\mathrm{H}$ in seals $[1,23]$.

Cyclospora cayetanensis, an emerging human pathogen, causes severe diarrheal disease and has resulted in several foodborne outbreaks in humans $[2,24,25]$. The transmission dynamics of this parasite are still unknown. In previous studies, feces as well as contaminated water sources were considered as transmission routes $[26,27]$.

Currently, no reports exist about simultaneous prevalence rates and genotyping data of cryptosporidiosis, microsporidiosis, giardiasis and cyclosporiasis in China. Therefore, this study focused on the prevalence and genetic characterizations of the four diseases in diarrhea outpatients of a pediatric clinic and an intestinal clinic in Shanghai and assessed their potential zoonotic transmission.

\section{Methods}

\section{Ethical statement}

Ethical clearance for the collection and examination of human feces samples was obtained from the Ethics Committee of the National Institute of Parasitic Diseases, Chinese Center for Disease Control and Prevention, China (reference no. 2012-12). The objectives, procedures and potential risk were orally explained to all participants. Written informed consent was given to, and signed by all participating in the study. Parents/guardians provided consent on behalf of all infant participants.

\section{Specimen collection and DNA extraction}

Fecal specimens from 252 diarrhea patients in a pediatric clinic $(n=169)$ and an intestinal clinic $(n=83)$ of a hospital in Pudong, Shanghai, China, were collected between October 2012 and March 2013. Patient details, including their age, gender, address, frequency of diarrhea and consistency of stools, were recorded. Specimens were collected from patients with fecal excretion heavier than $200 \mathrm{~g}$ and with no less than three events of diarrhea per day. The stools consistency was usually thin and mixed with mucus or blood. Sufficient samples were collected for DNA extraction and purification using the QIAamp DNA stool Mini Kit (QIAGEN, Hilden, Germany). The extracted DNA was stored at $-30^{\circ} \mathrm{C}$ for polymerase chain reaction $(\mathrm{PCR})$.

\section{Parasite identification in clinical samples}

The small subunit (SSU) rRNA gene of Cryptosporidium was identified using a nested PCR [28]. The presence of E. bieneusi, G. intestinalis and Cyclospora in the specimens was detected using individual nested PCR and sequence analysis of the SSU rRNA gene [29], the triose phosphate isomerase (TPI) gene [30] and the 18S rRNA gene [31], respectively.

All primers used in the study are listed in Table 1. Go $\mathrm{Taq}^{\circledR}$ Green Master Mix (containing Go Taq ${ }^{\bullet}$ DNA Polymerase, dNTP mixture, Green Go Taq Reaction Buffer, $\mathrm{MgCl}_{2}$; Promega) was used to amplify the genes of Cryptosporidium and G. intestinalis, while Premix Taq ${ }^{\circ}$ (containing Taq DNA Polymerase, dNTP mixture, Taq Buffer, Tartrazine/Xylene Cyanol FF; Takara) was used to identify Enterocytozoon and Cyclospora genes. Each $25 \mu \mathrm{l}$ reaction mixture contained $12.5 \mu \mathrm{l}$ Taq mix, $1 \mu \mathrm{l}$ of $10 \mu \mathrm{M}$ sense and antisense primers each, $1 \mu \mathrm{l}$ DNA template and $12.5 \mu \mathrm{l}$ nuclease-free water.

The thermal profile of Cryptosporidium PCR consisted of $94^{\circ} \mathrm{C}$ for $1 \mathrm{~min}, 35 \mathrm{cycles}$ of $94^{\circ} \mathrm{C}$ for $50 \mathrm{~s}, 55^{\circ} \mathrm{C}$ for $30 \mathrm{~s}$ and $72^{\circ} \mathrm{C}$ for $1 \mathrm{~min}$, followed by $72^{\circ} \mathrm{C}$ for $10 \mathrm{~min}$, with a hold step at $4^{\circ} \mathrm{C}$. A second reaction was carried out similarly. Each specimen was analyzed at least three times by PCR with positive and negative controls in each run. The other amplification conditions varied in annealing temperature and extension time. For Enterocytozoon, the annealing step was at $57.4^{\circ} \mathrm{C}$, and the extension step was at $72^{\circ} \mathrm{C}$ for $90 \mathrm{~s}$; the annealing step for Giardia was at $57.5^{\circ} \mathrm{C}$, and the extension step was at $72^{\circ} \mathrm{C}$ for $1 \mathrm{~min}$. The cycling conditions for Cyclospora were as follows: the primary cycle consisted of $94^{\circ} \mathrm{C}$ for $1 \mathrm{~min}, 35$ cycles of $94^{\circ} \mathrm{C}$ for $50 \mathrm{~s}, 56^{\circ} \mathrm{C}$ for $30 \mathrm{~s}$ and $72^{\circ} \mathrm{C}$ for $90 \mathrm{~s}$, followed by $72^{\circ} \mathrm{C}$ for $10 \mathrm{~min}$, and termination at $4^{\circ} \mathrm{C}$. The secondary step differed in extension time $\left(72^{\circ} \mathrm{C}\right.$ for $\left.1 \mathrm{~min}\right)$.

\section{Sequencing of each gene}

Secondary PCR products were directly sequenced on an ABI 3730 DNA Analyzer (Applied Biosystems, Foster City, USA) using the secondary primers and a Big Dye Terminator v3.1 Cycle Sequencing kit (Applied Biosystems). The sequence accuracy was confirmed by twodirectional sequencing and by sequencing a new PCR product if necessary. 
Table 1 Primers used for protozoan gene amplification

\begin{tabular}{|c|c|c|c|}
\hline Genus & Gene & Sequence of primers $\left(5^{\prime} \rightarrow 3^{\prime}\right)$ & Amplicon size (bp) \\
\hline \multirow[t]{4}{*}{ Cryptosporidium } & SSUrRNA & CryF1: TTCTAGAGCTAATACATGCG & $\sim 1325$ \\
\hline & & CryR1: CCATTTCCTTCGAAACAGGA & \\
\hline & & CryF2: GGAAGGGTTGTATTTATTAGATAAAG & $\sim 840$ \\
\hline & & CryR2: CTCATAAGGTGCTGAAGGAGTA & \\
\hline \multirow[t]{4}{*}{ Microsporidia } & SSUrRNA & EF1: GATGGTCATAGGGATGAAGAGCTT & $\sim 1200$ \\
\hline & & ER1: AATACAGGATCACTTGGATCCGT & \\
\hline & & EF2: AGGGATGAAGAGCTTCGGCTCTG & $\sim 600$ \\
\hline & & ER2: AATATCCCTAATACAGGATCACT & \\
\hline \multirow[t]{4}{*}{ Giardia } & TPI & TPIF1: AAATIATGCCTGCTCGTCG & $\sim 605$ \\
\hline & & TPIR1: CAAACCTTITCCGCAAACC & \\
\hline & & TPIF2: CCCTTCATCGGIGGTAACTT & $\sim 530$ \\
\hline & & TPIR2: GTGGCCACCACICCCGTGCC & \\
\hline \multirow[t]{4}{*}{ Cyclospora } & 18SrRNA & CYCLF1: AATGTAAAACCCTTCCAGAGTAAC & $\sim 1000$ \\
\hline & & CYCLR1: GCAATA ATCTATCCCCATCACG & \\
\hline & & CYCLF2: AATTCCAGCTCCAATAGTGTAT & $\sim 500$ \\
\hline & & CYCLR2: CAGGAGAAGCCAAGGTAGGCRTTT & \\
\hline
\end{tabular}

\section{Statistical analysis}

ContigExpress was used to assemble sequences. Sequences were aligned using the program ClustalX 1.83 (ftp://ftp-igbmc.u-strasbg.fr/pub/ClustalX/). All statistical analyses were performed using SPSS version 17.0 (SPSS Inc., Chicago, IL). The chi-squqre test was used to analyse the data, with $P<0.05$ considered to indicate significant differences.

\section{Nucleotide sequence accession numbers}

Representative nucleotide sequences were deposited in GenBank under the accession numbers KF271440 to KF271519.

\section{Results}

Occurrence of Cryptosporidium, Enterocytozoon, Giardia and Cyclospora

Cryptosporidium spp., E. bieneusi and G. intestinalis were detected by nested PCR in 34 (13.49\%), 34 (13.49\%) and 17 (6.75\%) of the 252 fecal specimens, respectively (Table 2).

Table 2 Sex ratio of Cryptosporidium, Enterocytozoon, Giardia and Cyclospora

\begin{tabular}{lllll}
\hline Genus & \multicolumn{4}{l}{ Number of positive specimens (\%) } \\
\cline { 2 - 5 } & Male & Female & Total & Species/genotype \\
\hline Cryptosporidium & $23(15.13)$ & $11(10.00)$ & $34(13.49)$ & C. andersoni \\
Enterocytozoon & $22(14.47)$ & $12(12.00)$ & $34(13.49)$ & - \\
Giardia & $11(7.24)$ & $6(6.00)$ & $17(6.75)$ & Assemblage C (16) \\
Cyclospora & 0 & 0 & 0 & Assemblage B (1) \\
Total patients & 152 & 100 & 252 & \\
\hline
\end{tabular}

Cyclospora was not detected. The Cryptosporidium-, E. bieneusi- and Giardia-positive patients were not restricted to a particular clinic. Polyparasitism was observed in nine of the 252 patients, eight of which were co-infected with Cryptosporidium and Enterocytozoon, while one was coinfected with Cryptosporidium and Giardia. No ageassociated differences in the patients involved (ranging from 1 month to 77 years) was found in our study.

\section{Cryptosporidium species}

Sequence analysis of Cryptosporidium indicated that all positive specimens belonged to $C$. andersoni, which is usually found in cattle. Patients tested in winter had a higher positivity rate $(17.31 \%)$ than those tested in spring (7.29\%, $P=0.024$, Figure 1$)$. However, no sex- or age-associated differences in detection rates were found $(P>0.05$; Figure 2$)$.

\section{Enterocytozoon infections}

Based on sequence analysis of the SSU rRNA gene, the microsporidia-positive outpatients were identified as $E$. bieneusi. Univariate analysis did not show any significant age- or sex-associated differences in E. bieneusi infection rates. However, patients showed higher detection rates for microsporidiosis in winter than in spring $(P<0.05$; Figure 1).

\section{Giardia genotyping}

Of the 17 Giardia-positive patients, most isolates belonged to assemblage $\mathrm{C}$, whereas only one belonged to assemblage $\mathrm{B}$; this result differs from previous reports 


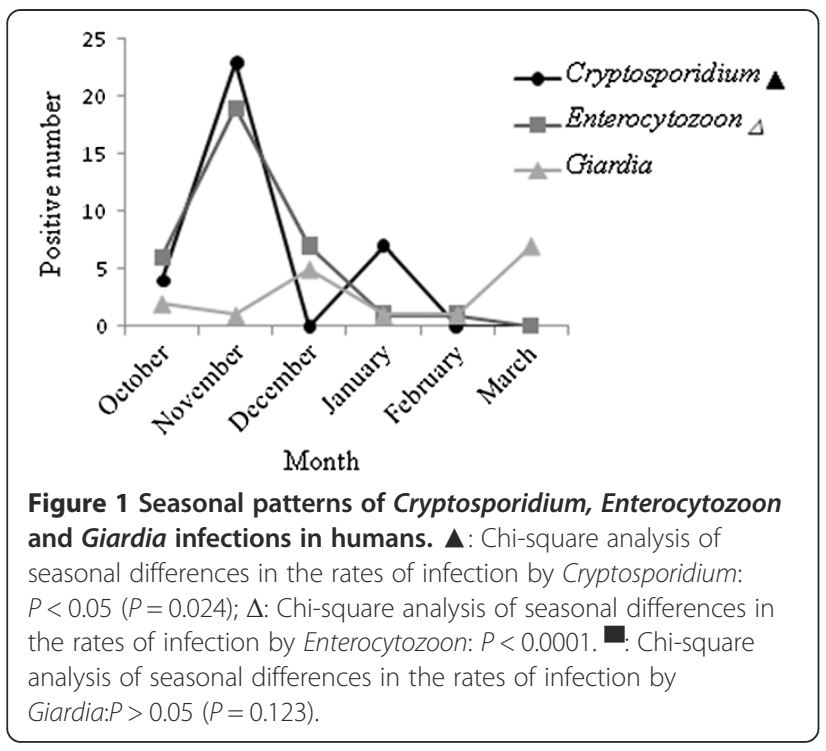

[4,21]. In addition, we found no differences associated with age, sex or season.

\section{Discussion}

Intestinal parasitic infections remain an important pathogenic factor of diarrhea in developing countries, especially among HIV-positive patients [32]. This study reports the epidemiological and genetic characterizations of four opportunistic intestinal parasites of outpatients from the pediatric clinic and intestinal clinic of a hospital in Shanghai, China. Using nested PCR and sequence analysis in this cross-sectional study, Cryptosporidium (13.49\%), Enterocytozoon (13.49\%) and Giardia (6.75\%) were possible etiologic agents in this area. However, Cyclospora was not detected in these patients. A high proportion (3.17\%) of polyparasitism was observed. The

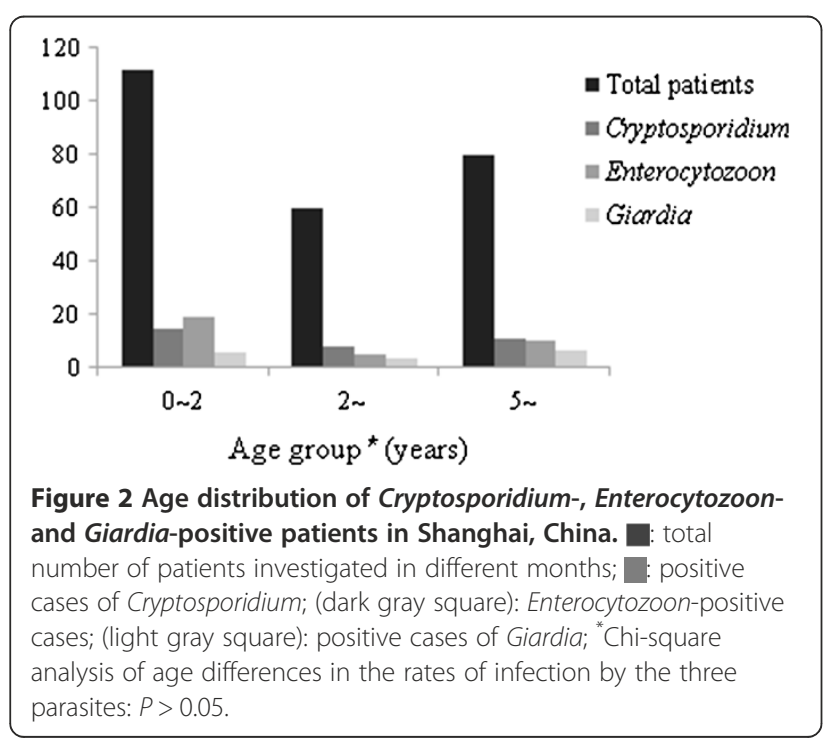

presence of co-infections was supported by the finding that these patients exhibited the most severe symptoms, with diarrhea frequencies reaching eight episodes per day.

C. andersoni is primarily found in cattle or in contaminated water [33,34], while few studies have reported $C$. andersoni infection in humans [15,35]. However, all Cryptosporidium-positive patients in this study were found to be infected with $C$. andersoni. The reasons for the occurrence of $C$. andersoni infection might be attributed to the environment, patient populations and geographic locations. Interestingly, our results were consistent with a recent study, which showed $C$. andersoni to be the dominant species in source and tap water in Shanghai [33]. Through genotyping, the researchers concluded that the contamination of source and tap water originated mainly from animal farms, especially, cattle farms. Similarly, C. andersoni was the most common species in the patients from our study, and sequence analysis suggested that contaminated water or infected cattles might be the possible transmission routes. In addition, the Yangtze River and Pudong Canal, which run through this area, are used for irrigation, livestock feeding and drinking water for residents. Extensive studies have reported seasonal differences in the distribution of C. parvum and C. hominis in the United Kingdom and New England, USA [36-38]. In Shanghai, increased C. andersoni detection rates during winter may have been due to changes in animal breeding, rainfall, travel and recreational activities.

Although $E$. bieneusi is nowadays considered to be an opportunistic pathogen in HIV-infected or organ transplant recipients, E. bieneusi infections have also been found in HIV-negative patients, immunocompetent and other healthy persons [39-42]. A recent study described a healthy man infected with a novel species of Microsporidium [43]. In the present study, 11 diarrheal adults (13.25\%) and 23 children (13.61\%) were detected with $E$. bieneusi, which suggested that this infection was not correlated with age in the study area $(P>0.05)$. However, humans were more susceptible to microsporidiosis in winter than in spring. The reason for this might be a reduced immunity and resistance during winter. Studies have reported E. bieneusi to be associated with acute and chronic diarrhea $[44,45]$. The sources of microsporidia infecting humans and its modes of transmission remain unclear. Due to the release of spores into the environment via stool and respiratory secretions, possible sources of infection might be humans or animals infected with microsporidia [4].

Genotyping results of G. intestinalis indicated that all but one positive specimens belonged to assemblage $C$, which is usually found in canids [46], thus suggesting that dogs may serve as sources of zoonotic transmission 
of giardiasis in this region. Sequence analyses of the TPI gene revealed that the only specimen from assemblage $B$ had a high homology with an isolate from a primate in Japan [47].

Overall, patients in Shanghai were found to be detected with C. andersoni, E. bieneusi and G. intestinalis. These three intestinal protozoa can be transmitted through the fecal-oral or oral-oral routes, inhalation of aerosols or ingestion of food or water contaminated with fecal material $[4,48]$. Therefore, it can be speculated that family members may also be infected, although more questionnaires and comprehensive epidemiological investigations are required to confirm this hypothesis. It has been reported that contamination of food or water by animals such as cattle or canids are causes of several foodborne and waterborne outbreaks of cryptosporidiosis [49-51]. In order to better understand the source of infection, we will attempt to seek the cooperation of patients involved in future studies to investigate their habits, such as contact with animals, drinking water and water conditions. In addition, we will continue to monitor the patients from the intestinal clinic and the pediatric clinic to determine whether the observed prevalence rates will persist. We also aim to extend the investigation to family members to determine the existence of a household cluster outbreak.

\section{Conclusions}

Based on our study, intestinal parasites were common among the study population of diarrheal outpatients in this area in Shanghai, China, between October 2012 and March 2013, C. andersoni, E. bieneusi and G. intestinalis (mainly assemblage $C$ ) were the major parasite species. The source of these infections remains to be tracked to determine their potential zoonotic transmission route.

\section{Competing interests}

The authors declare that they have no competing interests.

\section{Authors' contributions}

Conceived and designed the experiments: Y.S. J.C. H.L. Performed the experiments: HL YS JY ZY YX YJ WP YH Analyzed the data: YS HL JY JC Contributed reagents/materials/analysis tools: J.C. Y.S. Wrote the paper: HL YS JC. All authors read and approved the final version of the manuscript.

\section{Acknowledgments}

We thank Yifei Fu and Yi Fei (Shanghai Pudong District Center for Disease Control and Prevention), and the hospital personnel for assistance in specimen collection. We also thank Professor Longxian Zhang (College of Animal Science and Veterinary Medicine, Henan Agricultural University Zhengzhou, Henan, China) for kindly gift the positive samples of Enterocytozoon bieneusi as positive control. This work was supported by grants from the Shanghai Public Health Outstanding Academic Leader (No. GWDTR201214, to Y.S.), the National S \& T Major Program (Nos. 2012ZX10004-201 and 2013ZX10004805, to J.C.). Chinese Special Program for Scientific Research of Public Health (No. 201302004, to Y.S.). The funders had no role in study design, data collection and analysis, decision to publish, or preparation of the manuscript.
Received: 20 September 2013 Accepted: 2 January 2014

Published: 13 January 2014

\section{References}

1. Feng Y, Xiao L: Zoonotic potential and molecular epidemiology of Giardia species and giardiasis. Clin Microbiol Rev 2011, 24(1):110-140.

2. Ortega YR, Sanchez R: Update on Cyclospora cayetanensis, a food-borne and waterborne parasite. Clin Microbiol Rev 2010, 23(1):218-234.

3. Xiao L: Molecular epidemiology of cryptosporidiosis: an update. Exp Parasitol 2010, 124(1):80-89.

4. Mathis A, Weber R, Deplazes P: Zoonotic potential of the microsporidia. Clin Microbiol Rev 2005, 18(3):423-445.

5. Feng $Y$, Wang L, Duan L, Gomez-Puerta LA, Zhang L, Zhao X, Hu J, Zhang $\mathrm{N}$, Xiao L: Extended outbreak of cryptosporidiosis in a pediatric hospital China. Emerg Infect Dis 2012, 18(2):312-314.

6. Ye J, Xiao L, Ma J, Guo M, Liu L, Feng Y: Anthroponotic enteric parasites in monkeys in public park. China. Emerg Infect Dis 2012, 18(10):1640-1643.

7. Chalmers RM, Elwin K, Hadfield SJ, Robinson G: Sporadic human cryptosporidiosis caused by Cryptosporidium cuniculus, United Kingdom, 2007-2008. Emerg Infect Dis 2011, 17(3):536-538.

8. Giangaspero A, Berrilli F, Brandonisio O: Giardia and Cryptosporidium and public health: the epidemiological scenario from the Italian perspective. Parasitol Res 2007, 101(5):1169-1182.

9. Karanis $\mathrm{P}$, Kourenti $\mathrm{C}$, Smith $\mathrm{H}$ : Waterborne transmission of protozoan parasites: a worldwide review of outbreaks and lessons learnt. J Water Health 2007, 5(1):1-38.

10. Gostin LO, Lazzarini Z, Neslund VS, Osterholm MT: Water quality laws and waterborne diseases: Cryptosporidium and other emerging pathogens. Am J Public Health 2007, 90(6):847-853.

11. Crompton DW, Savioli L: Intestinal parasitic infections and urbanization. Bull World Health Organ 1993, 71(1):1-7.

12. Di Cristanziano V, Santoro M, Parisi F, Albonico M, Shaali MA, Di Cave D, Berrill F: Genetic characterization of Giardia duodenalis by sequence analysis in humans and animals in Pemba Island. Parasitol Int: Tanzania 2013:

13. Prado MS, Cairncross S, Strina A, Barreto ML, Oliveira-Assis AM, Rego S: Asymptomatic giardiasis and growth in young children:a longitudinal study in Salvador, Brazil. Parasitology 2005, 131:51-56.

14. Leoni F, Amar C, Nichols G, Pedraza-Díaz S, McLauchlin J: Genetic analysis of Cryptosporidium from 2414 humans with diarrhoea in England between 1985 and 2000. J Med Microbiol 2006, 55:703-707.

15. Gatei W, Ashford RW, Beeching NJ, Kamwati SK, Greensill J, Hart CA: Cryptosporidium muris infection in an HIV-infected adult. Kenya. Emerg Infect Dis 2002, 8(2):204-206.

16. Didier ES: Microsporidiosis: an emerging and opportunistic infection in humans and animals. Acta Trop 2005, 94(1):61-76.

17. Sulaiman IM, Fayer R, Lal AA, Trout JM, Schaefer FW 3rd, Xiao L: Molecular characterization of microsporidia indicates that wild mammals Harbor host-adapted Enterocytozoon spp. as well as human-pathogenic Enterocytozoon bieneusi. Appl Environ Microbiol 2003, 69(8):4495-4501.

18. Cama VA, Pearson J, Cabrera L, Pacheco L, Gilman R, Meyer S, Ortega Y, Xiao L: Transmission of Enterocytozoon bieneusi between a child and guinea pigs. J Clin Microbiol 2007, 45(8):2708-2710.

19. Monis PT, Andrews RH, Mayrhofer G, Ey PL: Genetic diversity within the morphological species Giardia intestinalis and its relationship to host origin. Infect Genet Evol 2003, 3(1):29-38.

20. Thompson RC, Hopkins RM, Homan WL: Nomenclature and genetic groupings of Giardia infecting mammals. Parasitol Today 2000, 16(5):210-213.

21. Thompson RC, Palmer CS, O'Handley R: The public health and clinical significance of Giardia and Cryptosporidium in domestic animals. Vet J 2008, 177(1):18-25.

22. Xiao L, Fayer R: Molecular characterisation of species and genotypes of Cryptosporidium and Giardia and assessment of zoonotic transmission. Int J Parasitol 2008, 38(11):1239-1255.

23. Cacciò SM, Ryan U: Molecular epidemiology of giardiasis. Mol Biochem Parasitol 2008, 160(2):75-80.

24. Bendall RP, Lucas S, Moody A, Tovey G, Chiodini PL: Diarrhoea associated with cyanobacterium-like bodies: a new coccidian enteritis of man. Lancet 1993, 341(8845):590-592.

25. Alfano-Sobsey EM, Eberhard ML, Seed JR, Weber DJ, Won KY, Nace EK, Moe CL: Human challenge pilot study with Cyclospora cayetanensis. Emerg Infect Dis 2004, 10(4):726-728. 
26. Chacin-Bonilla L: Transmission of Cyclospora cayetanensis infection: a review focusing on soil-borne cyclosporiasis. Trans $R$ Soc Trop Med Hyg 2008, 102(3):215-216.

27. Orlandi PA, Lampel KA: Extraction-free, filter-based template preparation for rapid and sensitive PCR detection of pathogenic parasitic protozoa. J Clin Microbiol 2000, 38(6):2271-2277.

28. Xiao L, Escalante L, Yang C, Sulaiman I, Escalante AA, Montali RJ, Fayer R, Lal AA: Phylogenetic analysis of Cryptosporidium parasites based on the small-subunit rRNA gene locus. Appl Env Microbiol 1999, 65(4):1578-1583.

29. da Silva AJ, Schwartz DA, Visvesvara GS, de Moura H, Slemenda SB, Pieniazek NJ: Sensitive PCR diagnosis of Infections by Enterocytozoon bieneusi (microsporidia) using primers based on the region coding for small-subunit rRNA. J Clin Microbiol 1996, 34(4):986-987.

30. Sulaiman IM, Fayer R, Bern C, Gilman RH, Trout JM, Schantz PM, Das P, Lal AA, Xiao L: Triosephosphate isomerase gene characterization and potential zoonotic transmission of Giardia duodenalis. Emerg Infect Dis 2003, 9(11):1444-1452

31. Dixon BR, Bussey JM, Parrington LJ, Parenteau M: Detection of Cyclospora cayetanensis oocysts in human fecal specimens by flow cytometry. J Clin Microbiol 2005, 43(5):2375-2379.

32. Mbae CK, Nokes J, Mulinge E, Nyambura J, Waruru A, Kariuki S: Intestinal parasitic infections in children presenting with diarrhoea in outpatient and inpatient settings in an informal settlement of Nairobi. Kenya. BMC Infect Dis 2013, 13(1):243

33. Feng $Y$, Zhao X, Chen J, Jin W, Zhou X, Li N, Wang L, Xiao L: Occurrence, source, and human infection potential of Cryptosporidium and Giardia spp. in source and tap water in shanghai, china. Appl Environ Microbiol 2011, 77(11):3609-3616.

34. Lindsay DS, Upton SJ, Owens DS, Morgan UM, Mead JR, Blagburn BL: Cryptosporidium andersoni n. sp. (Apicomplexa: Cryptosporiidae) from cattle, Bos taurus. J Eukaryot Microbiol 2000, 47(1):91-95.

35. Morse TD, Nichols RA, Grimason AM, Campbell BM, Tembo KC, Smith HV: Incidence of cryptosporidiosis species in paediatric patients in Malawi. Epidemiol Infect 2007, 135(8):1307-1315.

36. Learmonth JJ, Ionas G, Ebbett KA, Kwan ES: Genetic characterization and transmission cycles of Cryptosporidium species isolated from humans in New Zealand. Appl Environ Microbiol 2004, 70(7):3973-3978.

37. Hunter PR, Hughes S, Woodhouse S, Syed Q, Verlander NQ, Chalmers RM, Morgan K, Nichols G, Beeching N, Osborn K: Sporadic cryptosporidiosis casecontrol study with genotyping. Emerg Infect Dis 2004, 10(7):1241-1249.

38. McLauchlin J, Amar C, Pedraza-Díaz S, Nichols GL: Molecular epidemiological analysis of Cryptosporidium spp. in the United Kingdom: results of genotyping Cryptosporidium spp. in 1,705 fecal samples from humans and 105 fecal samples from livestock animals. J Clin Microbiol 2000, 38(11):3984-3990.

39. Goetz M, Eichenlaub S, Pape GR, Hoffmann RM: Chronic diarrhea as a result of intestinal microsposidiosis in a liver transplant recipient. Transplantation 2001, 71(2):334-337.

40. Metge S, Van Nhieu JT, Dahmane D, Grimbert P, Foulet F, Sarfati C, Bretagne S: A case of Enterocytozoon bieneusi infection in an HIV-negative renal transplant recipient. Eur J Clin Microbiol Infect Dis 2000, 19(3):221-223.

41. Albrecht $\mathrm{H}$, Sobottka I: Enterocytozoon bieneusi infection in patients who are not infected with human immunodeficiency virus. Clin Infect Dis 1997, 25(2):344.

42. Wanke CA, DeGirolami P, Federman M: Enterocytozoon bieneusi infection and diarrheal disease in patients who were not infected with human immunodeficiency virus: case report and review. Clin Infect Dis 1996, 23(4):816-818.

43. Suankratay C, Thiansukhon E, Nilaratanakul V, Putaporntip C, Jongwutiwes S: Disseminated infection caused by novel species of Microsporidium. Thailand. Emerg Infect Dis 2012, 18(2):302-304.

44. Subrungruang I, Mungthin $M$, Chavalitshewinkoon-Petmitr P, Rangsin R, Naaglor T, Leelayoova S: Evaluation of DNA extraction and PCR methods for detection of Enterocytozoon bienuesi in stool specimens. J Clin Microbiol 2004, 42(8):3490-3494.

45. Müller A, Bialek R, Kämper A, Fätkenheuer G, Salzberger B, Franzen C Detection of microsporidia in travelers with diarrhea. J Clin Microbiol 2001, 39(4):1630-1632

46. Beck R, Sprong H, Pozio E, Cacciò SM: Genotyping Giardia duodenalis Isolates from Dogs: lessons from a Multilocus Sequence Typing Study. Vector Borne Zoonotic Dis 2012, 12(3):206-213.
47. Suzuki J, Murata R, Kobayashi S, Sadamasu K, Kai A, Takeuchi T: Risk of human infection with Giardia duodenalis from cats in Japan and genotyping of the isolates to assess the route of infection in cats. Parasitology 2011, 138(4):493-500.

48. Eisenberg JN, Brookhart MA, Rice G, Brown M, Colford JM Jr: Disease transmission models for public health decision making: analysis of epidemic and endemic conditions caused by waterborne pathogens. Environ Health Perspect 2002, 110(8):783-790.

49. Blackburn BG, Mazurek JM, Hlavsa M, Park J, Tillapaw M, Parrish M, Salehi E, Franks W, Koch E, Smith F, Xiao L, Arrowood M, Hill V, da Silva A, Johnston S, Jones JL: Cryptosporidiosis associated with ozonated apple cider. Emerg Infect Dis 2006, 12(4):684-686.

50. Shukla R, Giraldo P, Kraliz A, Finnigan M, Sanchez AL: Cryptosporidium spp. and other zoonotic enteric parasites in a sample of domestic dogs and cats in the Niagara region of Ontario. Can Vet J 2006, 47(12):1179-1184.

51. Glaberman S, Moore JE, Lowery CJ, Chalmers RM, Sulaiman I, Elwin K, Rooney PJ, Millar BC, Dooley JS, Lal AA, Xiao L: Three drinking-waterassociated cryptosporidiosis outbreaks. Northern Ireland. Emerg Infect Dis 2002, 8(6):631-633.

doi:10.1186/1471-2334-14-25

Cite this article as: Liu et al:: Prevalence and genetic characterization of Cryptosporidium, Enterocytozoon, Giardia and Cyclospora in diarrheal outpatients in china. BMC Infectious Diseases 2014 14:25.

\section{Submit your next manuscript to BioMed Central and take full advantage of:}

- Convenient online submission

- Thorough peer review

- No space constraints or color figure charges

- Immediate publication on acceptance

- Inclusion in PubMed, CAS, Scopus and Google Scholar

- Research which is freely available for redistribution 